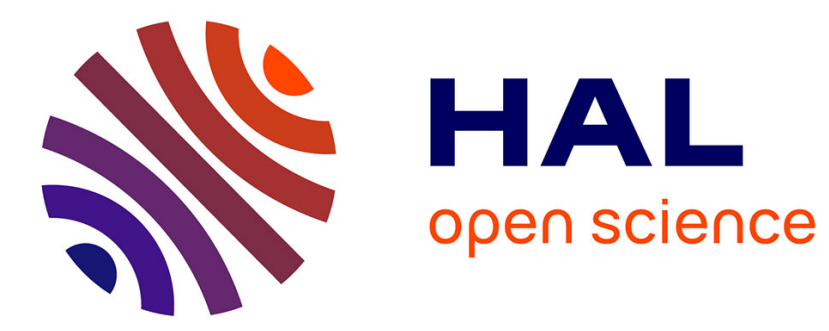

\title{
Centroid-based single sink placement in wireless sensor networks
}

Lemia Louail, Violeta Felea

\section{To cite this version:}

Lemia Louail, Violeta Felea. Centroid-based single sink placement in wireless sensor networks. Wireless Personal Communications, 2019, 108 (1), pp.121 - 140. hal-03221883

\section{HAL Id: hal-03221883 \\ https://hal.science/hal-03221883}

Submitted on 10 May 2021

HAL is a multi-disciplinary open access archive for the deposit and dissemination of scientific research documents, whether they are published or not. The documents may come from teaching and research institutions in France or abroad, or from public or private research centers.
L'archive ouverte pluridisciplinaire HAL, est destinée au dépôt et à la diffusion de documents scientifiques de niveau recherche, publiés ou non, émanant des établissements d'enseignement et de recherche français ou étrangers, des laboratoires publics ou privés. 


\title{
Centroid-based single sink placement in wireless sensor networks
}

\author{
Lemia Louail • Violeta Felea
}

Received: date / Accepted: date

\begin{abstract}
In wireless sensor networks, all collected data is sent to the sink through multi-hop communication and thus many performance metrics can be affected by the position of that sink especially when the deployment area contains empty zones where nodes cannot be deployed. In this paper, we present a new centroid-based single sink placement technique which exploits information about the shape of the deployment area and the shapes of the empty zones. The position of the single sink is computed using the positions of the centroids of the different geometric shapes in the deployment area. This computed position places the single sink as close as possible, in terms of geographic distance, to every node of the network in order to minimize the latency of communications. Simulation results show improvement of the average latency of communication and the average energy consumption using the centroid-based single sink placement technique compared to existing works.
\end{abstract}

Keywords Wireless sensor networks · Single sink placement · Centroids · empty zones

\section{Introduction}

Wireless sensor networks are used nowadays in many domains (healthcare, home networking, military, construction and manufacturing industries) in order to track certain features in the environment. These networks are composed of a large number of intelligent sensor nodes and one or many base stations also called sinks.

Lemia Louail

LRSD Laboratory, Faculty of Sciences, University of Sétif 1, Algeria

E-mail: lemia.louail@univ-setif.dz

Violeta Felea

Femto-st Institute/DISC, University of Bourgogne-Franche-Comté, France

E-mail: violeta.felea@femto-st.fr 
The sensor nodes are small electronic devices with limited resources (energy, memory, processing capacity); they are deployed in the environment to collect particular information and relay it, through multi-hop communication, to the sink.

The sink can be a sensor node or a computer, its main job is to receive data collected by the sensor nodes of the network and to transmit it to the final user.

Although many works are interested in finding the shortest path, in terms of geographic distance for instance, or the suitable MAC protocol to reduce energy consumption, they completely neglect the importance of the topology of the network. One of the main characteristics of wireless sensor networks is that many performance metrics, such as the latency of communication and the energy consumption, depend on the placement of the sink to which all collected data are addressed. If the sink is placed far from the nodes, this distance will induce more latency and energy consumption than if the sink were placed among the nodes.

The position of a single sink inside the network is an important issue on which we focus in this work, and more particularly in the case of a deployment area containing empty zones. We define an empty zone as a region where nodes can not physically be deployed because of obstacles, physical deterrents or any other physical constraints (see Figure 1).

In such a case the topology of the network is less regular and the sink needs to be placed so that it is as close as possible, in terms of geographic distance, to every node in the network, in order to minimize the latency of communication.

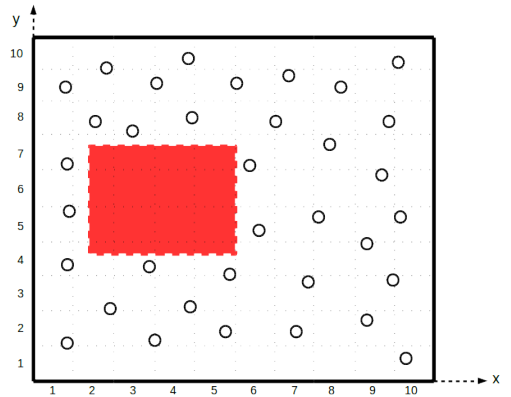

Fig. 1 An example of a deployment area for sensor networks containing an empty zone

Our main objective is to find a suitable position for a single sink in a wireless sensor network before exploiting the network. We use information about the deployment area, its shape and the shape of the empty zones. On the basis of this data, we propose a centroid method exploiting geometric shapes in order to place the sink.

The remainder of the paper is organized as follows. Section 2 presents some sink placement techniques existing in literature. In section 3 we present our centroid-based sink placement technique and in section 4 we evaluate the performances of our proposed sink placement technique compared to existing similar works. We finally conclude giving the advantages of our contribution. 


\section{Related works}

The position of the sink in the network affects directly many metrics such as the latency of the communication, the energy consumption of the nodes and the network lifetime. Therefore, several works proposed sink placement techniques or algorithms. Some of these works consider one sink while others are interested in placing several sinks.

The algorithm proposed in [1] tests all possible positions for the sink in the deployment area in order to find the position for which the first node to die in the network is the most recent.

Another work that proposes a complete traversal of the deployment area is presented in [3]; its idea is to select one target metric, the latency for example, and then to test every possible position for the sink while computing the metric. The position with the best value for the metric is selected as a permanent position for the sink.

The authors in [2] propose an algorithm that tries to determine a circle with the smallest diameter that encloses all the nodes. This circle can be formed, with at most three nodes, selected among the sensors of the network. The sink is positioned at the center of the circle. The evaluated metric in this work is the network lifetime i.e. the moment when the first node runs out of

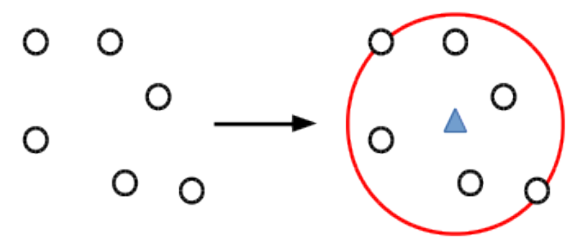

Fig. 2 Placing the sink in the center of the smallest enclosing circle for 6 nodes [2] energy.

The idea of [4] is to divide the deployment area into grids. The sink will be placed in the center of the grid with the maximum number of nodes. This work is also extended in order to place $k$ sinks in the $k$ most dense grids of the area. This placement of the sink allows it to have a maximum number of neighbours; these neighbours are used more than the other nodes of the network since they relay all the data coming from the network to the sink. Having more nodes in the neighbourhood of the sink reduces the energy consumption in the network [4].

Some works in literature propose the placement of several sinks while others place one or many mobile sinks [5-13]. Since we are interested in placing one fixed sink, we do not detail these works.

Table 1 summarizes the existing works, the number of sinks to place, the mobility of the sink and the target metric. 


\begin{tabular}{|c|c|c|c|}
\hline Paper & number of sinks & sink position & target metric \\
\hline$[1]$ & single & fixed & network lifetime \\
\hline [2] & single & fixed & network lifetime \\
\hline [3] & single & fixed & / \\
\hline [4] & single/multiple & fixed & energy consumption \\
\hline [5] & single/multiple & fixed & network lifetime \\
\hline [6] & single & fixed & distance between cluster heads and the sink \\
\hline$[7]$ & single & mobile & energy consumption/end to end delay \\
\hline [8] & single & mobile & $\begin{array}{c}\text { physical security } \\
\end{array}$ \\
\hline [9] & multiple & fixed & energy consumption \\
\hline [10] & multiple & fixed & energy consumption \\
\hline$[11]$ & multiple & fixed & percentage of dead nodes \\
\hline$[12]$ & multiple & fixed & latency/fault tolerance \\
\hline$[13]$ & multiple & fixed & energy consumtpion \\
\hline
\end{tabular}

Table 1 Existing sink placement approaches

All the existing works dealing with sink placement consider that the sensors can be positioned anywhere on the deployment area. This assumption may not always be confirmed because of several constraints, mostly imposed by the physical nature of the deployment area.

\section{Centroid-based single sink placement}

In this work we propose a centroid-based single sink placement technique in a deployment area containing empty zones. Our idea is to directly compute the position of the sink by exploiting information about the deployment area shape and the shapes of the empty zones.

In reality, a large deployment area can contain zones where nodes cannot be deployed. When deploying nodes in a large forest, for instance, in order to monitor temperature, there might be lakes or buildings in which nodes cannot be positioned. In such a case, the deployment area is not entirely accessible to sink placement, therefore, the sink position needs to be judiciously computed. The latency of communication is distance sensitive, thus our goal is to minimize it by placing the sink as close as possible, in terms of geographic distance, to every node in the network.

We assume that the sensor nodes are uniformly distributed in a square deployment area outside of the empty zones.

We note that this work does not focus on how to detect an empty zone in the deployment area; therefore, we suppose that the empty zones, their positions and their shapes can be identified by the administrator of the deployment area. Consequently, the latter can give it as input to our method.

The empty zone can have an irregular shape (see the left part of Figure 3 ), but in order to simplify the idea, we consider it as a rectangular shape, as shown in the right part of Figure 3. We note that this shape can be replaced by other simple geometric shapes in order to form more accurately the empty zones (triangles, circles, squares, ...). 


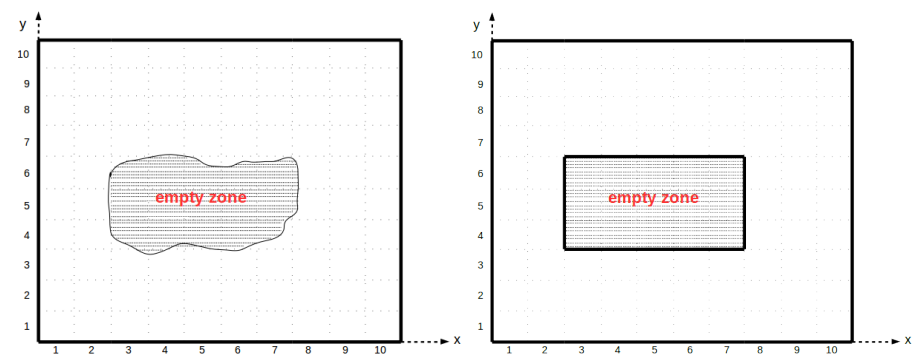

Fig. 3 A square deployment area containing an empty zone (left part) whose form is approximated by a rectangle (right part)

\subsection{Deployment area without empty zones}

When the deployment area does not contain any empty zones and nodes are uniformly distributed in it, the centroid of the square area is the nearest position to all other nodes of the network in terms of geographic distance. In such a case, the sink is directly placed in the centroid of the square area.

\subsection{Deployment area with empty zones}

In this section, we present our centroid-based technique in order to place the single sink in a deployment area containing empty zones. Our technique proceeds as follows:

1. We divide the deployment area into simple geometric shapes and we separate the empty zones from the deployment zones, the deployment zones are marked as $d z_{i}$ while the empty zones are marked as $e z_{i}$ where $i$ is the number of the area.

2. We find the centroid of each shape.

3. The position of the sink is computed using a weighted average of the centroids of the different geometric shapes and their area sizes such that deployment shapes are considered positively while empty zones shapes are considered negatively. The abscissa (x coordinate) of the sink is computed as following:

where:

$$
x_{\text {sink }}=\frac{\sum_{i=1}^{n} x d_{i} \times d z_{i}-\sum_{j=1}^{m} x e_{j} \times e z_{j}}{\sum_{i=1}^{n} d z_{i}-\sum_{j=1}^{m} e z_{j}}
$$

$n$ is the number of deployment zones,

$x d_{i}$ is the abscissa of the centroid of the deployment zone $i$,

$d z_{i}$ is the area size of the deployment zone $i$,

$m$ is the number of empty zones,

$x e_{j}$ is the abscissa of the centroid of the empimg2/ty zone $j$,

$e z_{j}$ is the area size of the empty zone $j$. 
The ordinate (y coordinate) of the sink is computed in the same way using the ordinates of the centroids:

$$
y_{\text {sink }}=\frac{\sum_{i=1}^{n} y d_{i} \times d z_{i}-\sum_{j=1}^{m} y e_{j} \times e z_{j}}{\sum_{i=1}^{n} d z_{i}-\sum_{j=1}^{m} e z_{j}}
$$

where:

$n$ is the number of deployment zones,

$y d_{i}$ is the ordinate of the centroid of the deployment zone $i$,

$d z_{i}$ is the area size of the deployment zone $i$,

$m$ is the number of empty zones,

$y e_{j}$ is the ordinate of the centroid of the empty zone $j$,

$e z_{j}$ is the area size of the empty zone $j$.

4. The sink is placed at the computed position.

We note that we only consider cases where the area size of the deployment zones is bigger than the area size of the empty zones, i.e. $\sum_{i=1}^{n} d z_{i}-\sum_{j=1}^{m} e z_{j}>0$. If not, we consider that the area is not suitable to deploy a connected sensor network.

Figure 4 shows an application example of our technique on a deployment area containing one empty zone.

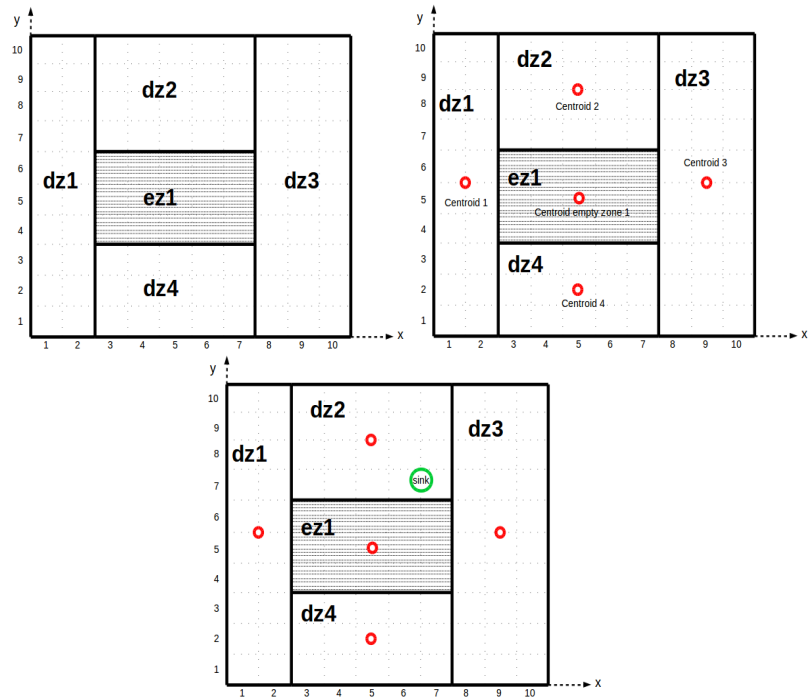

Fig. 4 left: Dividing the deployment area into simple geometric zones (step 1 of the technique), center: finding the centroid of each zone (step 2 of the technique), right: computing the position of the sink using equations 1 and 2 (step 3 and 4 of the technique) 
Figure 5 shows the same technique applied to a different placement of the empty zone into the deployment area. In this case it is placed in the bottom left corner of the area.

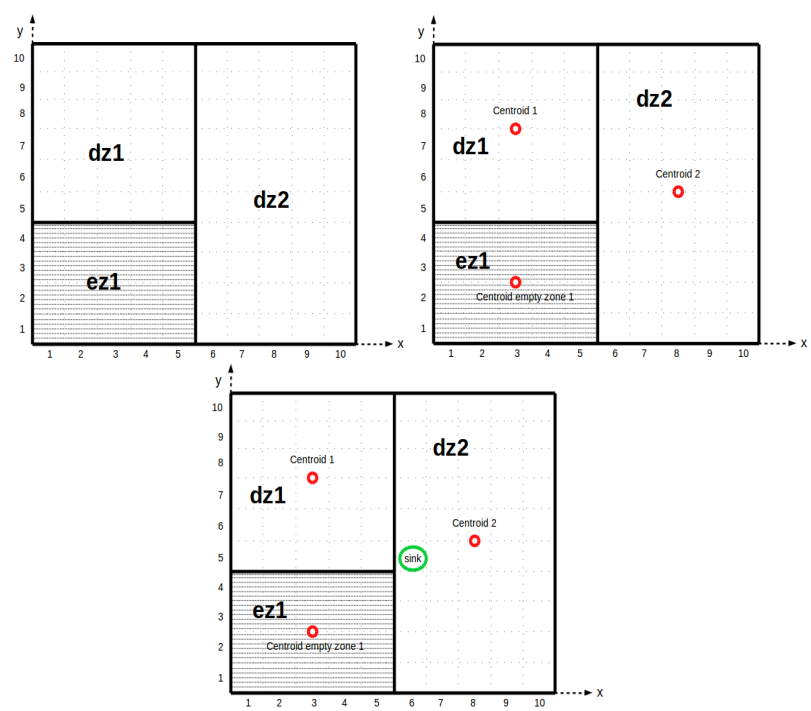

Fig. 5 Applying the centroid method on a deployment area with an empty zone in one corner

Figure 6 shows the application of the same technique on a deployment area containing two empty zones.

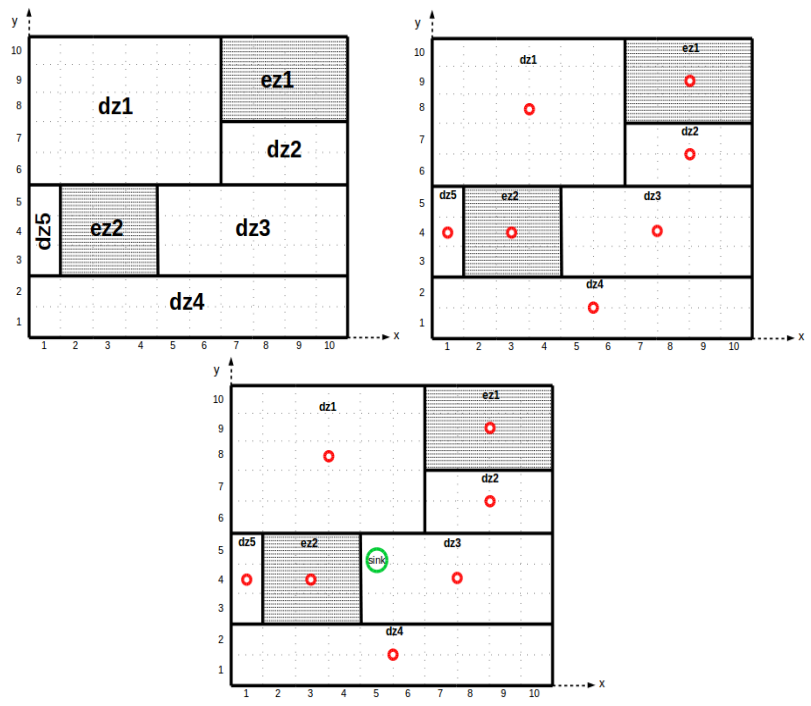

Fig. 6 Applying the centroid method on a deployment area with two empty zones 
3.3 Sink position adjustment

Our centroid method computes the position of the single sink based on the centroids of the different zones formed from the deployment area which contains one or several empty zones. But there is no guaranty that the computed position of the sink will not be in an empty zone. If that is the case, we propose to adjust the position of this sink in order to place it out of the empty zone.

For instance, if the empty zone is placed in the center of the deployment area, the final position of the sink computed with our centroid method will be in the exact position of the centroid of that empty zone shape (see the left and the center parts in Figure 7). In such a case, our solution is to move the sink to the nearest position in the deployment area outside of the empty zone. The empty circle is the adjusted position corresponding to the computed one (marked by a circle containing the sink label).

If the sink is placed in the center of the empty zone, four positions are possible for repositioning the sink as shown in the right part of Figure 7.

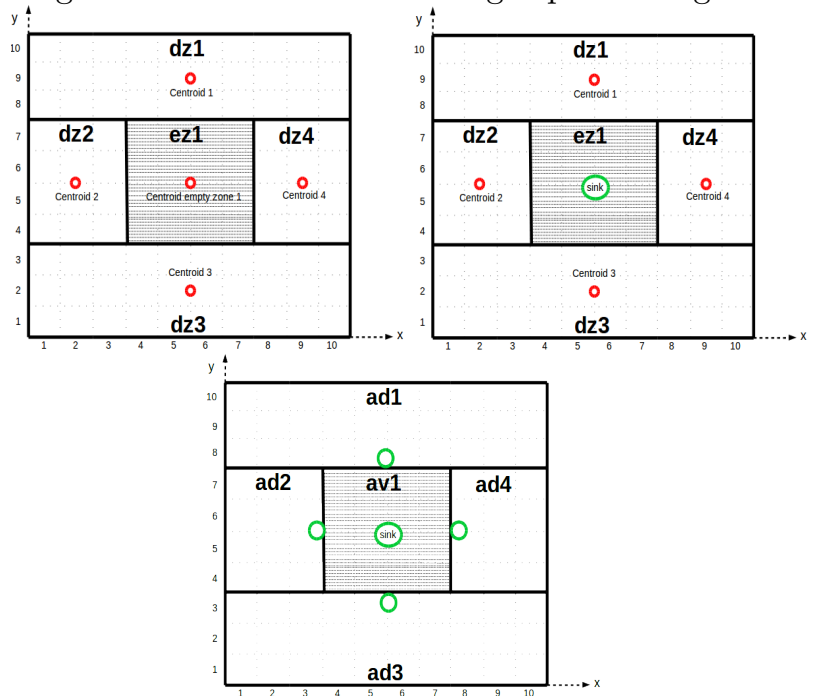

Fig. 7 Adjusted sink position when it is placed in the center of the empty zone

If the sink is placed in the empty zone but not in its center, we propose to adjust its computed position by moving it to the nearest location outside the empty zone as follows:

We note by

$x_{\text {right }}$ - the difference between the abscissa of the sink and the abscissa of the right side of the empty zone area,

$x_{\text {left }}$ - the difference between the abscissa of the sink and the abscissa of the left side of the empty zone area,

$y_{u p}$ - the difference between the ordinate of the sink and the ordinate of the upper side of the empty zone area,

$y_{\text {bottom }}$ - the difference between the ordinate of the sink and the ordinate of the bottom side of the empty zone area. 
New sink coordinates are computed as follows:

$$
\begin{aligned}
& x_{\text {sink }}=\left\{\begin{array}{c}
x_{\text {sink }}+x_{\text {right }} \text { if } x_{\text {right }}<x_{\text {left }} \\
x_{\text {sink }}-x_{\text {left }} \text { if } x_{\text {right }}>x_{\text {left }}
\end{array}\right. \\
& y_{\text {sink }}=\left\{\begin{array}{cl}
y_{\text {sink }}+y_{\text {up }} & \text { if } y_{\text {up }}<y_{\text {bottom }} \\
y_{\text {sink }}-y_{\text {bottom }} & \text { if } y_{\text {up }}>y_{\text {bottom }}
\end{array}\right.
\end{aligned}
$$

Figure 8 shows two examples of an adjusted sink position when its computed position is inside an empty zone.
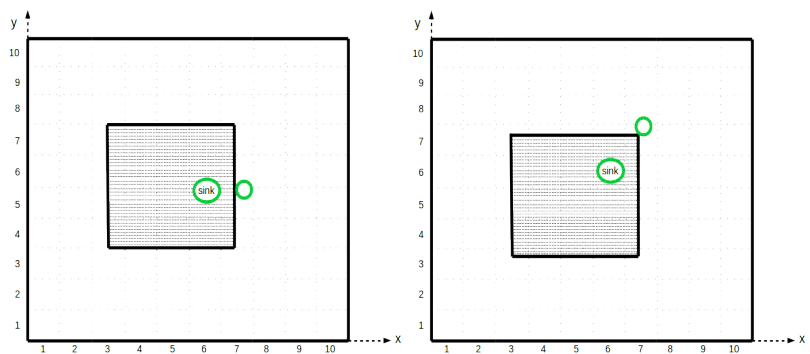

Fig. 8 Example of an adjusted sink position when the computed coordinates of the sink are inside an empty zone

Figure 9 (from left to right) shows an example of placing the sink in a deployment area with two empty zones. The sink position needs to be adjusted using equations 3 and 4 , if computations based on equations 1 and 2 place the sink in an empty zone.
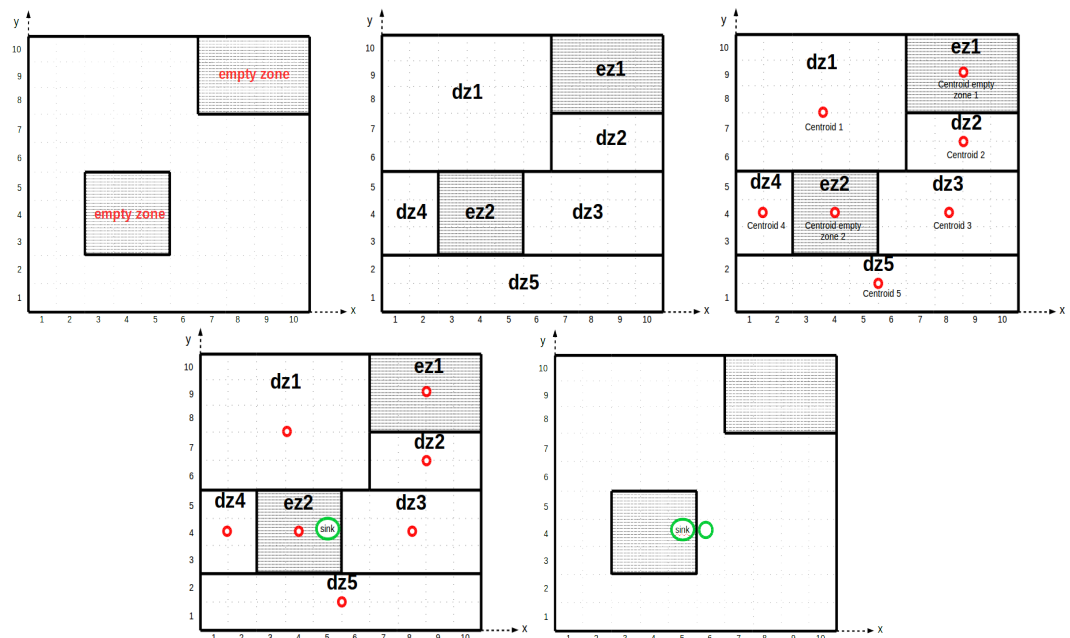

Fig. 9 Sink placement using the centroid technique in a deployment area containing two empty zones

\section{Evaluation and simulation results}

In order to evaluate our sink placement technique we compare it with three other techniques. The first one, that we denote by Circle, is proposed in [2]; 
the idea is to determine a circle with the least diameter that encloses all the nodes of the network and to place the sink in the center of that circle. The second one, that we denote by DenseGrid, is proposed in [4] which divides the deployment area into grids and then places the sink in the most dense grid. The last one proposed in [3], and denoted DeploymentAreaTraversal, uses a complete traversal of the deployment area in order to choose a suitable placement for the sink in terms of the target metric.

Performances of the approaches were simulated using JUNG [15], a Javabased library that allows modeling, analysis and visualization of networks as graphs. First, the deployment area of size $a^{2}$ is generated. After that, the empty zones are inserted in the deployment area. Finally, $n=100$ nodes are distributed uniformly in the deployment zones where each node has a communication range $r=25 \mathrm{~m}$. The connectivity model is UDG (Unit Disk Graph [16]), in which two nodes are considered neighbours if and only if they are within each other's transmission range $r$. A set of 50 networks was generated randomly using these parameters.

After placing the sink with each technique, simulations are launched where every node of the network has one packet to deliver to the sink.

A random TDMA schedule is used as a MAC protocol where each node has one slot to transmit data to its next hop and as many slots as the number of its neighbours to receive data from them. The TDMA was chosen because it ensures the absence of collisions in the network and the latency is simply computed in number of slots. A geographic routing is used as a routing protocol where each node chooses as its next hop one of its neighbours that is the closest in terms of geographic distance to the sink. Since the goal of this paper is not the evaluation of MAC or of routing protocols but the impact of the placement of the sink in the network, we note that any other MAC or routing protocol can be used.

For each one of the 50 simulations, one network is generated and the four sink placement techniques are applied. Each network simulation has its own TDMA schedule. The routing tree might be different since the position of the sink, which is the root of the tree, is not the same for each technique.

We evaluate two metrics:

- the average latency: it is the average of delivery times for every node (except for the sink). The delivery time for one node is computed as the number of slots needed for a packet generated by the node to arrive to the sink through multiple hops according to the routing tree information. It includes the waiting time before the node can send the packet during its sending slot and the time needed so that the packet arrives to the sink.

- the average energy consumption: it is the average of the energy consumption of all the nodes of the network (except for the sink). The energy consumption of a node is computed as in LEACH [17]: when a node sends 
a packet its energy is depleted with a value in transmission mode and its neighbours' energy is depleted with a value in reception mode.

The first test uses a deployment area with one empty zone in the bottom left corner. The sink's position obtained using the four techniques is shown in Figure 10.

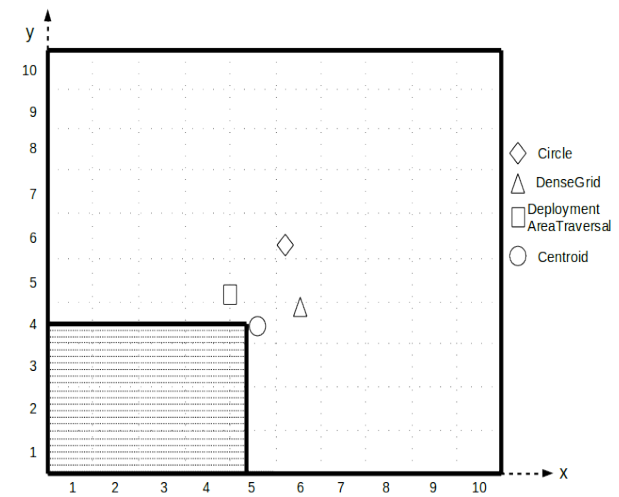

Fig. 10 An example of the different sink positions obtained by the four placement techniques

We give performances of our centroid placement technique compared to existing works in Figures 11 and 12.

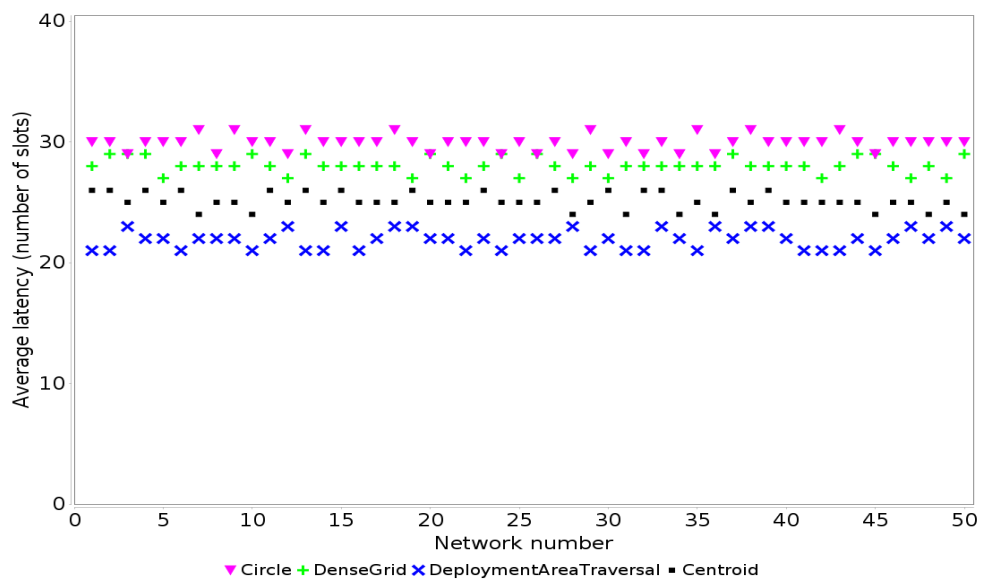

Fig. 11 Average latency for each network with one empty zone 


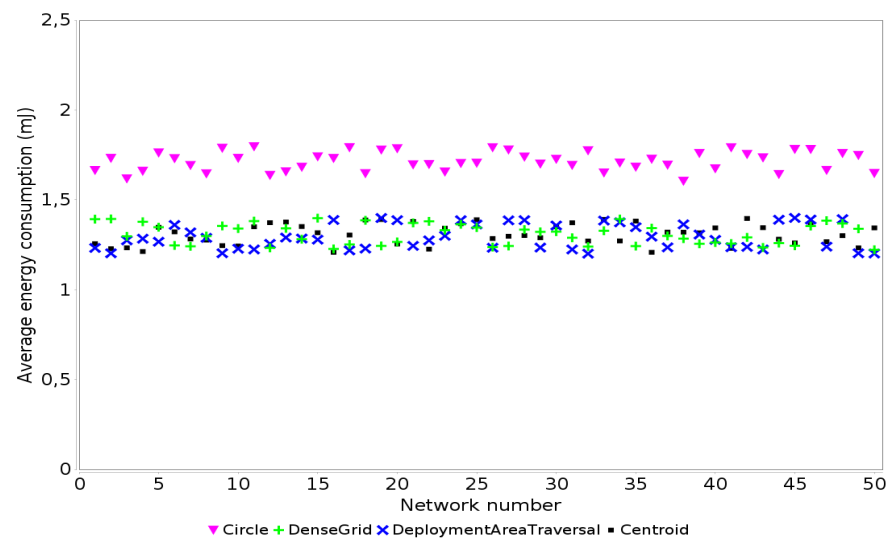

Fig. 12 Average energy consumption for each network with one empty zone

The DenseGrid technique places the sink in the most dense grid of the deployment area. This allows the sink to have more neighbors but does not guarantee that the sink will be closer to the other nodes of the network. Our centroid technique places the sink as close as possible, in terms of geographic distance, to every node in the network. Therefore, the centroid technique provides better average latency.

Regarding the energy consumption, the goal of the DenseGrid technique is to minimize the energy consumption of the nodes of the networks by positioning the sink in the most dense grid of the deployment area. This idea is based on the fact that the neighbors of the sink are used more frequently than the other nodes of the network because in addition to sending their data they must relay data coming from other nodes. This allows the sink to have many neighbours that can forward data towards it, but whenever one of these neighbours is sending data, the others are consuming their energy in the reception mode. On the other hand, our centroid technique is based on minimizing the distance between every node of the network and the sink. The two techniques experience approximately similar results in terms of energy consumption.

The DeploymentAreaTraversal technique allows looking for the best position for the sink in terms of average latency by testing every possible integer cartesian coordinate in the deployment area and comparing the latencies obtained by each position, therefore it has the optimal average latency. The limit of this technique is the long execution time it requires. For one simulation with 100 nodes, our Centroid technique execution takes less than 1 minute while The DeploymentAreaTraversal technique execution takes 7 minutes.

The Centorid tehcnique can not perform better than the Deployment area technique since the latter finds the optimal latency, but it can have close results in much less time. 
The Circle technique always places the sink in the center of the deployment area which, in the presence of empty zones, is not a suitable position. Indeed, it does not provide a good average latency or average energy consumption.

The previous results are averages of the obtained metrics for the 100 nodes in each generated network and do not reflect data individually. We analyze the extent of variability of the obtained values in relation to the correspondent mean, on the basis of the coefficient of variation (Relative Standard Deviation). The coefficient of variation is expressed in percentage as $\frac{\sigma}{\mu}$ where $\sigma$ is the standard deviation and $\mu$ is the mean. Table 2 shows the intervals for the coefficient of variation for the 50 generated networks. All the obtained deviations are inferior to $11.0 \%$ which shows that the absolute values are not very scattered.

\begin{tabular}{|c|c|c|}
\hline [CVmin ; CVmax $]$ & Latency & Energy consumption \\
\hline Circle & {$[8.2 ; 10.0] \%$} & {$[8.3 ; 10.6] \%$} \\
DenseGrid & {$[6.5 ; 10.3] \%$} & {$[7.0 ; 10.3] \%$} \\
DeploymentAreaTraversal & {$[7.6 ; 10.0] \%$} & {$[7.4 ; 11.0] \%$} \\
Centroid & {$[6.4 ; 10.2] \%$} & {$[7.3 ; 10.1] \%$} \\
\hline
\end{tabular}

Table 2 Intervals for the coefficient of variation concerning results of metrics when the network contains a single empty zone

Table 3 summarizes the results presented in Figures 11 and 12 . It presents the gains and the losses of the Centroid technique compared to the other sink placement techniques in terms of average latency and average energy consumption.

\begin{tabular}{|c|c|c|}
\hline & average latency & average energy consumption \\
\hline Circle [2] & $+15.8 \%$ & $+23.2 \%$ \\
\hline DenseGrid [4] & $+10.09 \%$ & $+0.5 \%$ \\
\hline DeploymentAreaTraversal [3] & $-13 \%$ & $-0.7 \%$ \\
\hline
\end{tabular}

Table 3 Gains (+) and losses (-) of the Centroid technique compared to existing works

Figure 13 shows an example of a deployment area with two empty zones and the placement of the sink with each technique.

We note that the Circle technique could not be used when one of the empty zones is in the center of the deployment area since it places the sink in the center of the empty zone. 


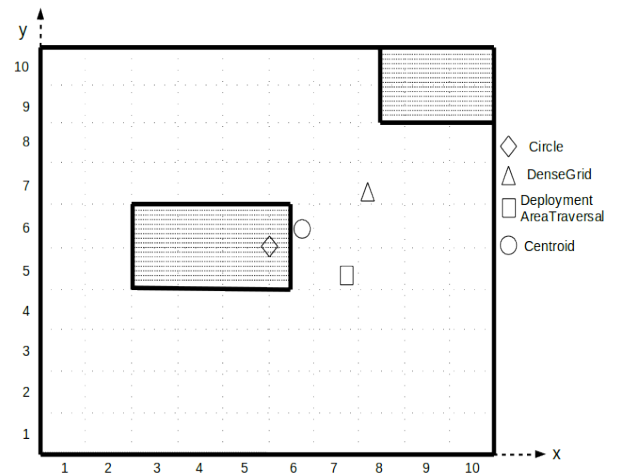

Fig. 13 An example of a deployment area with two empty zones and the different sink positions using the four placement techniques

Figures 14 and 15 give the results in terms of average latency and average energy consumption with the deployment area shown in Figure 13. The performances are similar to the previous ones.

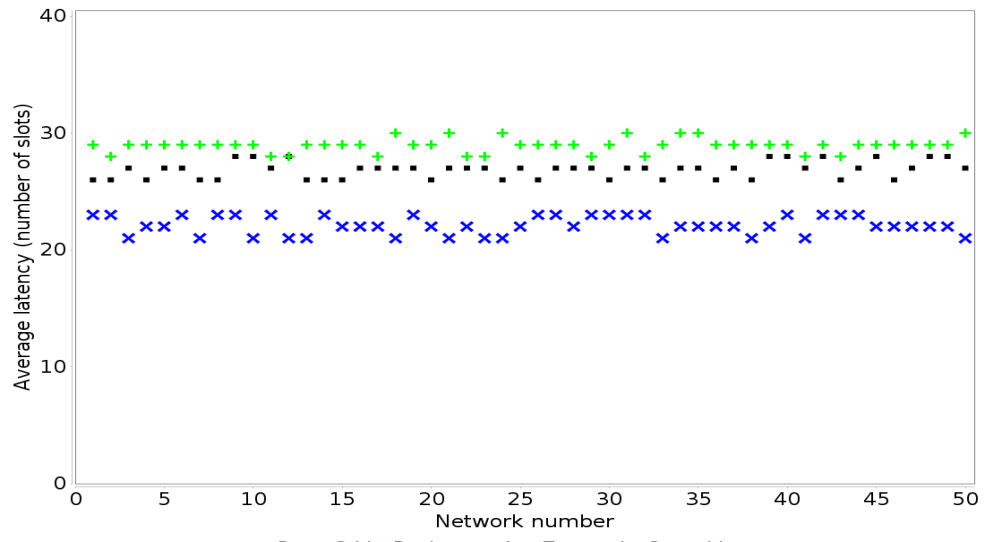

Fig. 14 Average latency of networks with two empty zones

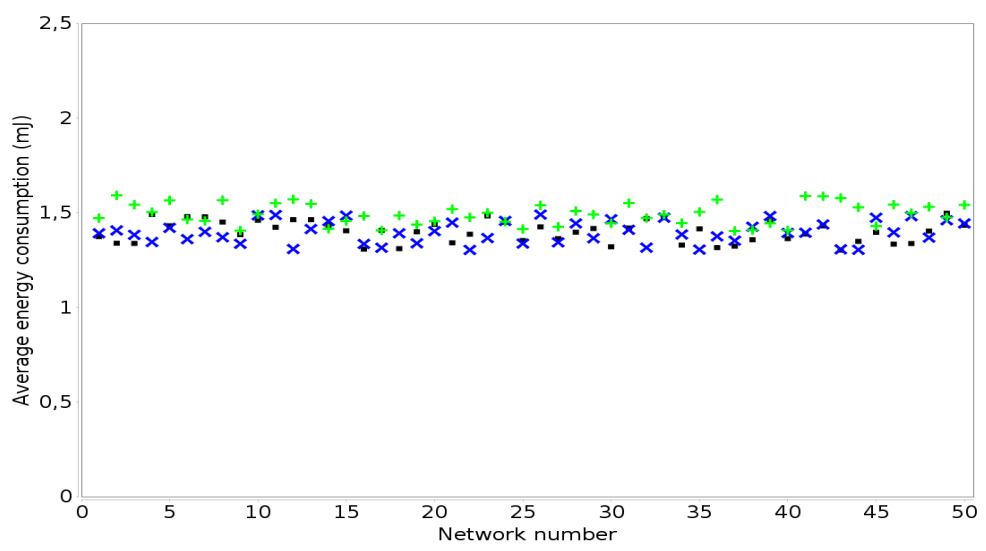

Fig. 15 Average energy consumption of networks with two empty zones 
Table 4 presents the coefficient of variation for the results concerning two empty zones. The obtained deviations are inferior to $11.1 \%$ which shows that the absolute values are not very scattered.

\begin{tabular}{|c|c|c|}
\hline [CVmin ; CVmax $]$ & Latency & Energy consumption \\
\hline DenseGrid & {$[7.2 ; 11.1] \%$} & {$[6.8 ; 9.8] \%$} \\
DeploymentAreaTraversal & {$[8.1 ; 10.6] \%$} & {$[7.6 ; 10.9] \%$} \\
Centroid & {$[7.3 ; 10.6] \%$} & {$[7.4 ; 10.2] \%$} \\
\hline
\end{tabular}

Table 4 Intervals for the coefficient of variation concerning results for networks with two empty zones

Table 5 summarizes the results presented in Figures 14 and 15. It presents the gains and the losses of the Centroid technique compared to the other sink placement techniques in terms of average latency and average energy consumption.

\begin{tabular}{|c|c|c|}
\hline & average latency & average energy consumption \\
\hline DenseGrid [4] & $+7.04 \%$ & $+6.61 \%$ \\
\hline DeploymentAreaTraversal [3] & $-18 \%$ & $-0.27 \%$ \\
\hline
\end{tabular}

Table 5 Gains $(+)$ and losses (-) of the Centroid technique compared to existing works

\section{Results on real data sets}

we selected a real data set to test our Centroid-based single sink placement technique.

As shown in Figure 16, 92 sensor nodes are deployed in the city of Lille (North of France) in order to monitor the level of water in this area.

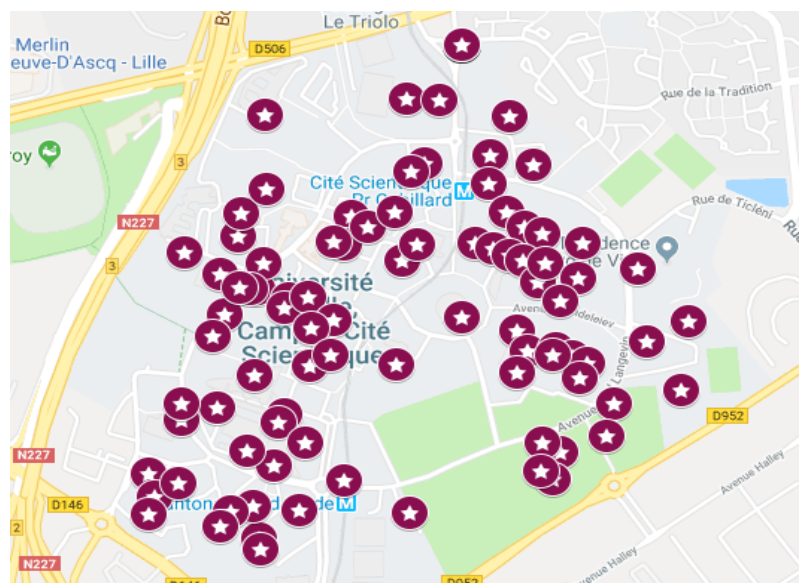

Fig. 1692 sensors deployed in Lille (North of France) to monitor the level of water 
The positions of these sensor nodes are shown in the Cartesian plane of Figure 17.

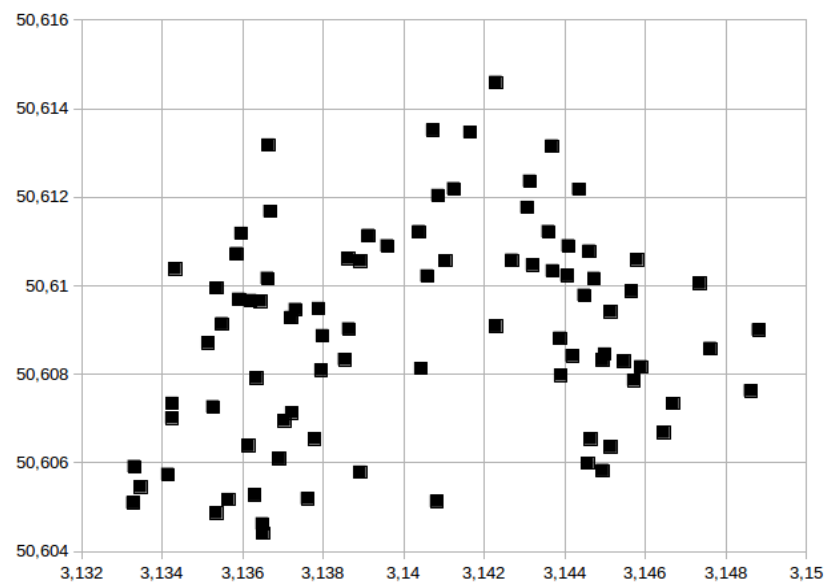

Fig. 17 The positions of the 92 sensors of Figure 16

This deployment area contains one empty zone where nodes are not deployed. We represent this empty zone by a red rectangular shape as shown in Figure 18.

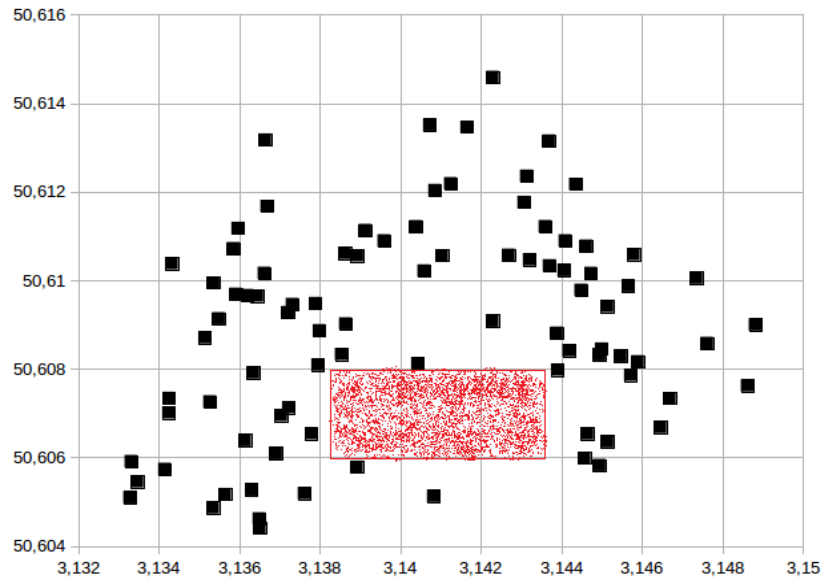

Fig. 18 An empty zone represented by a red rectangle

After that, we applied the four sink placement techniques cited in our paper to this deployment area: Circle, DenseGrid, DeploymentAreaTraversal and our technique Centroid. The four obtained placements are shown in Figure 19 


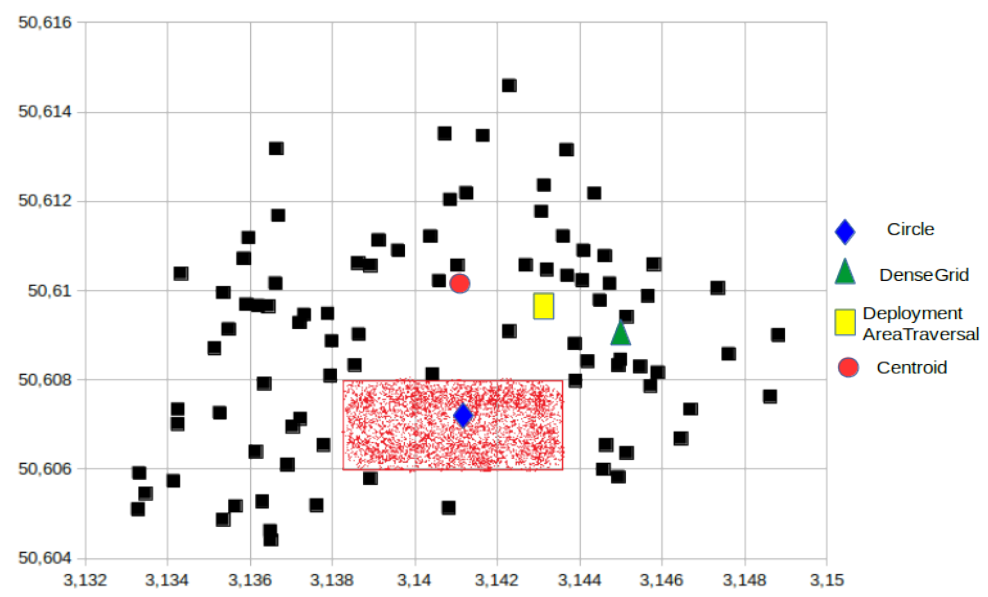

Fig. 19 Sink positions with the four techniques

We used the same protocols as in our previous simulations, i.e. a random TDMA as a MAC approach and a geographic routing as routing approach. The results, in terms of average latency and average energy consumption using each of the sink positions, are presented in Table 6 .

We note that there are no results for the Circle technique since its sink is placed in the empty zone.

\begin{tabular}{|c|c|c|}
\hline & Latency (number of slots) & Energy consumption $(\mathrm{mJ})$ \\
\hline Circle & $/$ & $/$ \\
DenseGrid & 26 & 1.33 \\
DeploymentAreaTraversal & 23 & 1.306 \\
Centroid & 24 & 1.225 \\
\hline
\end{tabular}

Table 6 Average latency and average energy consumption with different sink positions for the network of Figure 16

The obtained results are coherent with the results of our previous simulations. The Centroid method has a better latency than DenseGrid and is not far from the optimal latency.

A different deployment of the same 92 nodes is shown in Figure 20, in this case the deployment area contains two empty zones. 


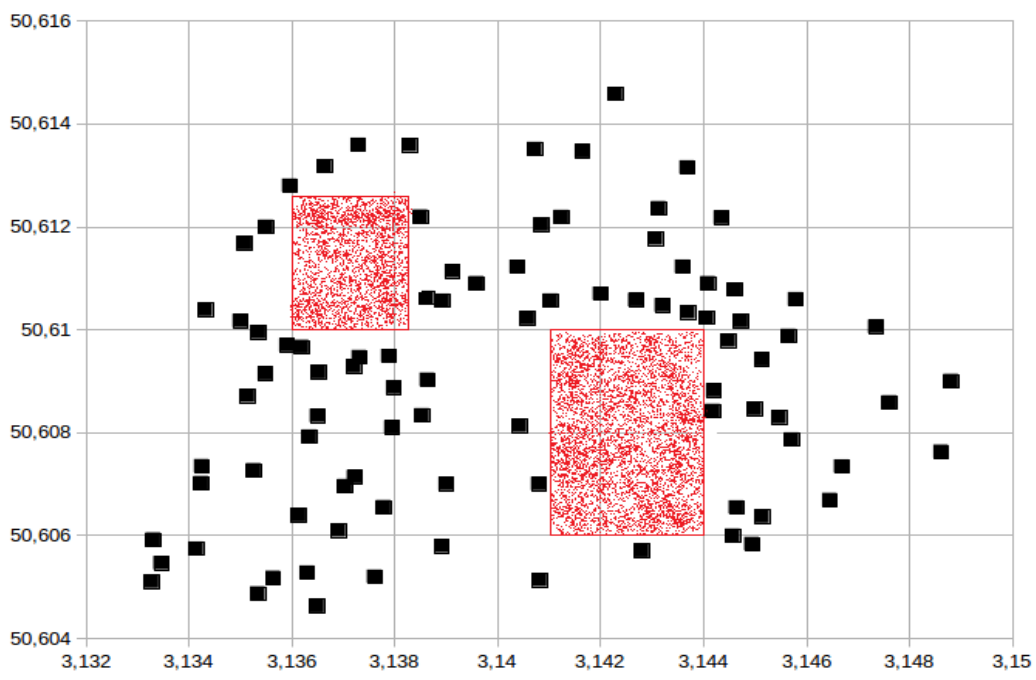

Fig. 20 A deployment Area with two empty zones

The positions of the sink using the four methods are presented in Figure 21

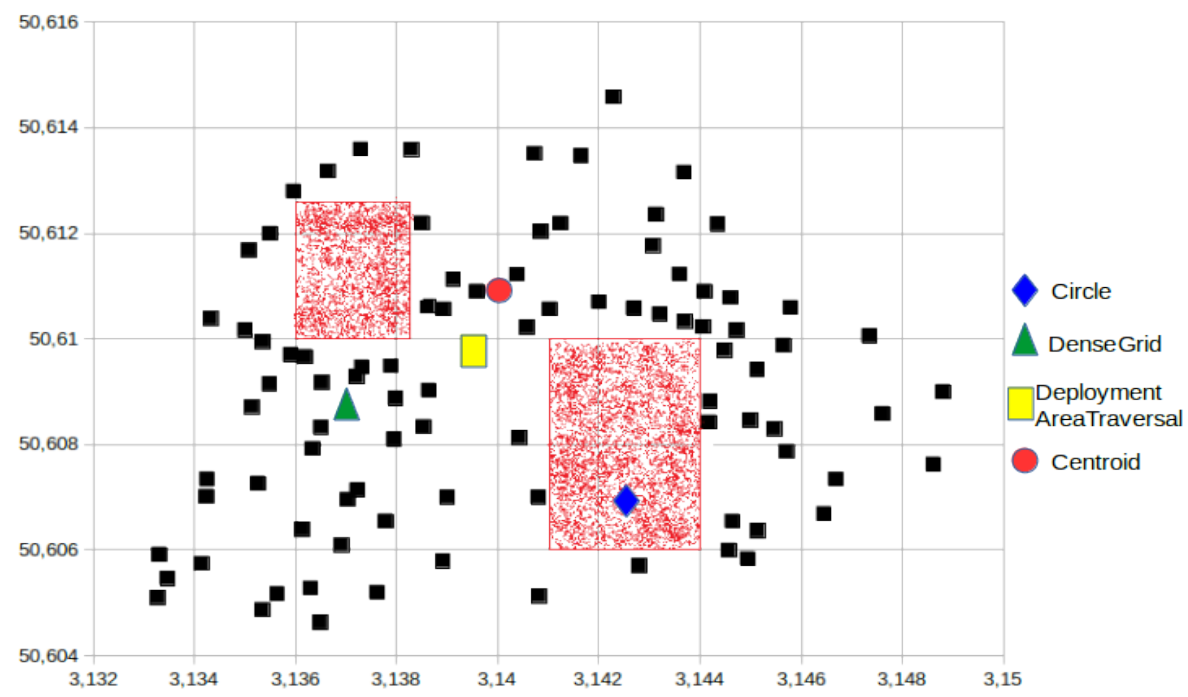

Fig. 21 Sink positions in the deployment Area with two empty zones

The results, in terms of average latency and average energy consumption using each of the sink positions, are presented in Table 7 . In this case, the Centroid method has also a better latency than the DenseGrid method and 
is not far from the optimal latency obtained by the DeploymentAreaTraversal method which is our goal.

\begin{tabular}{|c|c|c|}
\hline & Latency (number of slots) & Energy consumption (mJ) \\
\hline Circle & $/$ & $/$ \\
DenseGrid & 28 & 1.41 \\
DeploymentAreaTraversal & 25 & 1.5 \\
Centroid & 26 & 1.429 \\
\hline
\end{tabular}

Table 7 Average latency and average energy consumption with different sink positions for the network of Figure 20

\section{Conclusion}

In this paper, we presented a new Centroid-based single sink placement technique in order to place a single sink in a wireless sensor network. Our main objective is to minimize the latency of communication by placing the sink as close as possible, in terms of geographic distance, to every node of the network. Our technique uses information about the deployment area containing empty zones where nodes cannot be deployed. The idea is to exploit the shape of the deployment area as well as the shapes of the empty zones and then to compute the position of the sink based on the position of the centroids of the different shapes.

Results of different simulations showed improvement in terms of the average latency of communication and of the average energy consumption using the Centroid-based single sink placement technique compared to existing works.

\section{References}

1. Y. Shi and Y. T. Hou and A. Efrat, Algorithm design for base station placement problems in sensor networks, Proceedings of the 3rd international conference on QSHINE, page 13, 2006, ACM

2. J. Pan and L. Cai and Y. T. Hou and Y. Shi and S. X.Shen, Optimal base-station locations in two-tiered wireless sensor networks, IEEE Transactions on Mobile Computing, volume 4, number 5, pages 458-473, 2005

3. K. Akkaya and M. Younis and W. Youssef, Positioning of base stations in wireless sensor networks, IEEE Communications Magazine, volume 45, number 4, 2007

4. D. Das and Z. Rehena and S. Roy and N. Mukherjee, Multiple-sink placement strategies in wireless sensor networks, 5th International Conference COMSNETS, pages $1-7,2013$

5. J. Flathagen and O. Kure and P. E. Engelstad, Constrained-based multiple sink placement for wireless sensor networks, IEEE 8th International Conference on MASS, pages 783-788, 2011

6. B. Paul and M. A. Matin and M. J. Showkat and Z. Rahman, Optimal placement of base stations in a two tiered wireless sensor network, WSEAS Transactions on Communications, volume 1, 2010

7. K. Akkaya and M. Younis and M. Bangad, Sink repositioning for enhanced performance in wireless sensor networks, Computer Networks, Elsevier, volume 49, number 4, pages 512-534, 2005 
8. W. Youssef and M. Younis and K. Akkaya, An intelligent safety-aware gateway relocation scheme for wireless sensor networks, IEEE International Conference on Communications, volume 8, pages 3396-3401, 2006

9. P. González-Brevis and J. Gondzio and Y. Fan and H. V. Poor and J. Thompson and I. Krikidis and P. J. Chung, Base station location optimization for minimal energy consumption in wireless networks, IEEE 73rd VTC Spring, pages 1-5, 2011

10. A. Bogdanov and E. Maneva and S. Riesenfeld, Power-aware base station positioning for sensor networks, 23rd Annual Conference of the IEEE INFOCOM, volume 1, 2004

11. E. I. Oyman and C. Ersoy, Multiple sink network design problem in large scale wireless sensor networks, IEEE International Conference on Communications, volume 6, pages 3663-3667, 2004

12. M. M. S. Sarwar and P. Chatterjee, Optimal Sink Placement in Wireless Sensor Networks to Increase Network Performance, Industry Interactive Innovations in Science, Engineering and Technology, pages 423-433, Springer, 2018

13. P. Pardesi and J. Grover, Improved multiple sink placement strategy in wireless sensor networks, IEEE International Conference on Futuristic Trends on Computational Analysis and Knowledge Management (ABLAZE), pages 418-424, 2015

14. R. Beghdad and A. Lamraoui, Boundary and holes recognition in wireless sensor networks, Journal of Innovation in Digital Ecosystems, volume 3, n 1, pages 1-14, 2016

15. J. O'Madadhain and D. Fisher and P. Smyth and S. White and Y. Boey, Analysis and visualization of network data using JUNG, Journal of Statistical Software, volume 10, number 2, pages 1-35, 2005

16. S. Schmid and R. Wattenhofer, Algorithmic models for sensor networks, 14th WPDRTS, pages 450-459, 2006

17. W.R. Heinzelman and A. Chandrakasan and H. Balakrishnan, Energy-Efficient Communication Protocol for Wireless Microsensor Networks, HICSS, pages 3005-3014, 2000 